\title{
RESEARCH
}

Open Access

\section{Serum defensin levels in patients with systemic sclerosis}

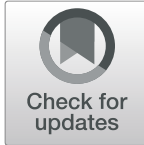

Tugce Emiroglu Gedik ${ }^{1,2^{*}}$ (D, Hamit Kucuk ${ }^{3}$, Berna Goker ${ }^{3}$, Seminur Haznedaroglư ${ }^{3}$, Hatice Pasaoglu4 , Ozkan Varan ${ }^{3}$, Mehmet Akif Ozturk ${ }^{3}$, Ozge Tugce Pasaoglu ${ }^{4}$ and Abdurrahman Tufan ${ }^{3}$

\begin{abstract}
Background: Systemic sclerosis (SSc) is an autoimmune disease characterized by fibrosis of skin and lung as well as involvement of kidney, gastrointestinal system and heart. Aetiology and exact mechanism of disease is poorly understood. The association between antimicrobial peptides (AMPs) and other diseases such as idiopathic pulmonary fibrosis, diffuse panbronchiolitis, pulmoner alveolar proteinosis and psoriasis have been reported. A small number of studies have examined the role of AMPs on autoimmune diseases which has not been studied in scleroderma yet. We aimed to investigate AMP serum levels and their association with disease characteristics of SSc.

Methods: Forty-two patients ( 40 female, mean age 42 years) and 38 healthy subjects (32 female, mean age 38 years) were enrolled. For SSc patients, the following data were recorded: disease subset (limited/diffuse), autoantibodies (antinuclear, anti-centromere (ACA), and anti-SCL-70), blood tests, erythrocyte sedimentation rate (ESR), and C-reactive protein (CRP), modified Rodnan skin score, presence and history of digital ulcers, kidney, gastrointestinal disease and lung involvement assessed by computed tomography and pulmonary function tests. Association between serum AMPs and disease characteristics were analysed.
\end{abstract}

Results: Twenty-nine of the patients had diffuse (69\%) and 13 of the patients had limited (31\%) systemic sclerosis. Average disease duration was 5.5 years. Pulmonary involvement was detected in 20 patients (47.6\%). Serum concentration of alpha defensin was higher than healthy subjects (563 $\pm 415 \mathrm{vs} 377 \pm 269 \mathrm{ng} / \mathrm{mL}, p=0.02$ ). However, no difference was observed for beta-1 and beta-2 defensins in SSc patients and healthy controls. In subgroup analysis patients with interstitial lung disease had higher levels of alpha defensin than those without lung involvement ( $684 \pm 473$ vs $430 \pm 299 \mathrm{ng} / \mathrm{ml}, p=0.04$ ). There was also correlation between alfa defensin serum concentrations and CRP $(r=0.34)$.

Conclusions: Alpha defensin levels are increased in scleroderma patients and correlated with lung involvement indicating a role in the pathogenesis of disease.

Trial registration: This study is not a clinical trial study.

Keywords: Systemic sclerosis, Alpha defensin, Beta defensin, Antimicrobial peptides, Pathogenesis, Inflammation

\footnotetext{
* Correspondence: tugceemiroglu@yahoo.com.tr

'Department of Internal Medicine, Gazi University Faculty of Medicine,

Ankara, Turkey

${ }^{2}$ Department of Internal Medicine, Division of Geriatrics, Istanbul

University-Cerrahpasa, Cerrahpasa Faculty of Medicine, Istanbul, Turkey

Full list of author information is available at the end of the article
}

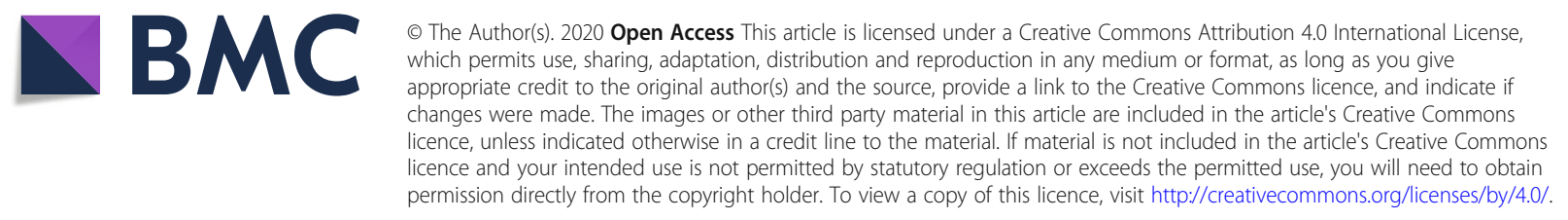




\section{Introduction}

Systemic sclerosis (SSc) is a chronic multisystemic autoimmune disease manifested by diffuse fibrosis of the skin and internal organs [1]. Although the pathogenesis of the disease is largely unknown, three major pathologic changes take part in its evolution; fibrosis, vasculopathy and inflammation. SSc is largely considered as an autoimmune disease with activation of both innate and adaptive components of immune system [2].

One of the critical components of innate immune system is antimicrobial peptides (AMPs). There are hundreds of structurally different peptides with antimicrobial properties which are found in all eukaryotic organisms. AMPs are particularly synthesized in mucosal epithelial cells and neutrophils that are constantly in contact with microorganisms in the body. In humans, there are three main family of AMPs; defensins, cathelicidin (LL37) and histatin [3]. Alpha-defensins (HNP1) are primarily expressed in neutrophils, while beta-defensins and LL37 are constitutively synthesized and secreted from epithelial surfaces, therefore called as epithelial AMPs [4]. AMPs have direct microbicidal effects and play critical role in cleaning intracellular microorganisms and fighting against cancer cells $[5,6]$. Besides having direct antimicrobial effects, AMPs can incite inflammation by activating immune effector cells and chemotaxis of neutrophils [4]. Epithelial AMPs may stimulate production of pro-inflammatory cytokines such as IL-17, IL-22, and TNF alpha [7]. Another important role of AMPs involves the maintenance of the host microbiota besides their antimicrobial functions.

To date, limited number of studies have investigated the relationship between antimicrobial peptides and autoimmune/inflammatory diseases. In patients with systemic lupus erythematosus (SLE), alpha defensin and beta-2 defensin levels were found elevated and related with disease activity $[8,9]$. There is not much literature on the potential role of AMPs in SjS; however, some studies suggest that cathelicidin and defensins may have a role in the pathophysiology of the disease [10]. The role of AMPs in pulmonary diseases have been shown in idiopathic pulmonary fibrosis, diffuse panbronchiolitis and pulmonary alveolar proteinosis. The role of AMPs are scarcely studied in scleroderma and there is downregulation of LL-37 in diffuse SSc patients [11] and tissue beta defensin of localized scleroderma after UVA-1 treatment [12]. Herein, we aimed to investigate alpha and beta defensin serum concentrations in patients with SSc and their association with disease characteristics.

\section{Materials and methods}

The study included consecutive SSc patients over 18 years of age who met the diagnostic criteria for SSc [13]. Patients with other autoimmune disease or overlap syndromes, infections, immunodeficiency, chronic liver and kidney dysfunction were excluded from the study. Patients receiving corticosteroids and immunosuppressive drugs were excluded from the study. Control group consisted age- and sex-matched healthy subjects. Written consent was obtained and included in the study. All participants were informed about the objectives of the study and gave their signed consent for inclusion. The study was approved by the local ethics committee (Ethics committee approval ID: 28.09.2015/3).

Demographic and clinical features of SSc patients were obtained by patient interviews and hospital file records. Detailed physical examination was performed for each patient and skin fibrosis was assessed by an experienced physician. Laboratory parameters, acute phase reactants and serologic tests including anti-nuclear antibodies, anti-scl-70 and anti-centromere antibodies were retrieved from computer based hospital records. Pulmonary function tests, high resolution thorax computed tomography (HRCT) and echocardiographic findings were recorded. Interstitial lung disease was defined as interstitial fibrosis, reticulation or alveolitis on highresolution computer tomography. Pulmonary hypertension was defined as mean pulmonary artery pressure $\geq$ $25 \mathrm{~mm} / \mathrm{Hg}$. Gastrointestinal involvement was evaluated in all subjects with oesophageal manometry and considered positive if there was consistent findings. Finally, SSc patients were categorized by LeRoy's classification system as diffuse and limited SSc [14].

Blood samples were taken from antecubital vein after overnight fasting at morning hours and immediately centrifuged. Sera was separated and stored at $-80^{\circ} \mathrm{C}$ until they studied. Serum alpha defensin (HNP1), betadefensin 1 and 2 levels were determined by using commercial enzyme-linked immunosorbent assay (ELISA) kits (Assay Biotech, USA).

Statistical Package for the Social Sciences software v16.0 (SPSS Inc., Chicago, IL) was used for statistical analysis. Categorical data were expressed as numbers and percentages. Chi square test was used to compare categorical variables. Normality distribution of continuous variables were evaluated with using KolmogorovSmirnov test. Continuous variables are expressed as median [Interquartile range (IQR)] values since they did not show normal distribution. Continuous variables of groups were compared with Mann-Whitney $\mathrm{U}$ and Kruskal-Wallis tests. Spearman Rho was used for correlation analyzes. Differences between groups were considered significant at the $5 \%$ level $(p<0.05)$.

\section{Results}

A total of 80 individuals, 42 SSc patients and 38 healthy control subjects were included in the study. Forty patients were female and mean age of patients was $42 \pm 11$ 
Table 1 Clinical manifestations of systemic sclerosis patients

\begin{tabular}{ll}
\hline Clinical manifestations & N (\%) \\
\hline Lung Involvement & $20(47.6)$ \\
Pulmonary hypertension & $11(31.4)$ \\
Esophageal involvement & $13(31)$ \\
Intestinal involvement & $2(4.8)$ \\
Renal involvement & $2(4.8)$ \\
Telangiectasia & $20(48.8)$ \\
Raynaud's phenomenon & $41(97.6)$ \\
Digital ulcer & $16(36.8)$ \\
Autoantibodies & \\
ANA & $42(100)$ \\
Anti-Scl-70 & $27(67.5)$ \\
Anti- centromere & $10(25)$ \\
RNP & 0 \\
SS-A & $9(25.7)$ \\
SS-B & $2(5.7)$ \\
\hline
\end{tabular}

years. Mean age of control group was $38 \pm 11$ years and 32 were female.

Twenty-nine $(69 \%)$ patients had diffuse and 13 patients $(31 \%)$ had limited systemic sclerosis. The mean disease duration was $5.5 \pm 4.8$ years. Organ involvements of the patients are presented in Table 1 . Pulmonary involvement on HRCT was evident in $20(47.6 \%)$ patients.

Serum alpha defensin levels of SSc patients were significantly higher than control subjects $(563 \pm 415$ vs $377 \pm 269 \mathrm{ng} / \mathrm{mL}, p=0.02$ ). However no differences were observed in serum concentrations of epithelial defensins - beta-1 and beta-2 between SSc patients with healthy subjects (beta- 1 defensin $235 \pm 178$ vs $185 \pm 24 \mathrm{pg} / \mathrm{mL}$, $p=0.08$ and beta- 2 defensin $253 \pm 453$ vs $152 \pm 101 \mathrm{pg} /$ $\mathrm{ml}, p=0.1$. There were no statistically significant differences in alpha, beta- 1 and beta- 2 defensin levels between diffuse and limited forms of SSc ( $p=0.08, p=0.56, p=$ 0.57 ; respectively). In sub-group analysis, with regard to organ involvements, patients with interstitial lung disease had remarkably higher levels of alpha defensins than those without lung involvement $(684 \pm 473$ vs
$430 \pm 299 \mathrm{ng} / \mathrm{mL}, p=0.04$, Table 2 ). However any association was found between organ manifestations and serum concentrations of epithelial defensins - beta-1 and beta- 2 in patients with SSc. There was a correlation between CRP $(r=0.345, p=0.03)$, modified Rodnan Score $(r=0.296 p=0.07)$ and $\%$ forced vital capacity FVC $(r=0.306 p=0.07)$.

\section{Discussion}

In this cross-sectional study, we investigated serum levels of the one of the major antimicrobial peptide family, defensins. We found that serum concentrations of alpha defensins are increased in SSc patients. Moreover, elevation in serum alpha defensin (HNP- $\alpha$ ) was more remarkable in SSc patients with pulmonary involvement.

AMPs are part of the innate immunity with capability of activating adaptive immune system [15]. The level of AMP expression varies among individuals, and it has been suggested that this variation is due to genetic differences in the genes encoding defensins. These peptides are also known as danger signals or" alarmins" playing important roles in inflammation and immunity [16]. AMPs can be released into the extracellular area following granulocyte activation as a consequence of degranulation, cell death, leakage, and lysis during inflammation [17]. Recent studies show that the abnormal production of AMPs produced by neutrophils or epithelial cells promotes inflammation by supporting the autoimmune response. However, there are not enough studies related to the significance of abnormal levels of AMPs in SSc.

AMP levels have been studied in some inflammatory diseases such as rheumatoid arthritis, SLE, inflammatory bowel disease, psoriasis, atopic dermatitis and familial Mediterranean fever (FMF). These studies showed that AMPs expression were irregular in diseases such as systemic lupus erythematosus (SLE), psoriasis, rheumatoid arthritis (RA), type 1 diabetes (T1D), Sjögren's disease (SjS) and multiple sclerosis (MS) [10]. Hiroyuki Tamiya et al. reported significantly higher defensin and cathelicidin levels in active SLE patients [18]. Tufan et al. found similar results in FMF patients that increased alphadefensin and cathelicidin levels with no significant

Table 2 Alpha defensin levels according to clinical manifestations of systemic sclerosis

\begin{tabular}{llll}
\hline Clinical manifestations & No involvement & Involvement present & $P$ values \\
\hline Interstitial lung disease & $430[140-595](n=22)$ & $684[324-1152](n=20)$ & $0.04^{*}$ \\
Pulmonary hypertension & $503[139-888](n=31)$ & $624[300-991](n=11)$ & 0.4 \\
Raynaud's phenomenon & $512(n=1)$ & $564[157-890](n=41)$ & 0.95 \\
Telangiectasia & $621[388-894](n=22)$ & $498[135-879](n=20)$ & 0.29 \\
Digital Ulcer & $583[165-938](n=26)$ & $529[164-813](n=16)$ & 0.56 \\
Esophageal Involvement & $588[352-890](n=29)$ & $506[124-933](n=13)$ & 0.34 \\
\hline
\end{tabular}

Values represent median with 25-75 percentiles in square brackets and the number of patients in parentheses

${ }^{*} p$ value $<0,05$ 
difference for beta-2-defensin [19]. In another study on psoriasis patients, use of serum beta-2-defensin levels are suggested as a marker reflecting psoriasis disease activity [20]. Cathelicidin and human beta-defensins 2 and 3 (hBD2/3) have been shown to be highly expressed in the psoriatic skin of patients [21,22]. The expression of human neutrophil peptides (HNPs) 1-3 was significantly increased in the synovial cavity of patients with RA and related with joint erosion [23].

Previous studies have demonstrated increased AMP levels in inflammatory lung diseases. It was shown that HNP levels were 50 times higher in bronchoalveolar lavage samples of ARDS patients [24]. In a study by Noriho Sakamoto et al., AMP levels were studied in patients with interstitial pneumonia (IP). Their study included 21 acute attack IP, 44 stable IP, 9 infected IP patients and 23 healthy controls. The level of alpha-defensin was found to be significantly increased in patients with acute exacerbation. Beta- defensin- 2 was found to be higher in patients with stable IP than in healthy controls [25]. Mukae et al. found that serum alpha-defensin levels were higher in patients with idiopatic pulmonary fibrosis (IPF) compared to control group. But no difference was found between bronchoalveolar lavage levels [26]. In another study, Mukae et al. compared defensin levels of IPF, cryptogenic organized pneumonia (COP), pulmonary alveolar proteinosis PAP), sarcoidosis, and idiopathic nonspecific interstitial pneumonia (NSIP) patients and defensin levels were significantly higher than the control group. There was a significant difference between the diseases as well. Beta defensin-1 levels were significantly higher in IPF, COP, NSIP and sarcoidosis patients compared to the control group. Beta-2 defensin levels were significantly higher in sarcoidosis and IPF patients compared to the control group [27].

Defensin family AMPs have not been thoroughly studied in patients with SSc. In our study, we aimed to determine serum levels of main defensins and their association with disease characteristics. We found significantly increased serum alpha defensin levels particularly in those with interstitial lung involvement. Alphadefensin is mainly released from neutrophils and activate neutrophils with a positive feedback loop and they might contribute lung inflammation observed in SSc and other pathologies. Supporting this hypothesis, serum alpha defensin levels were correlated with acute phase reactants. Interestingly, serum alpha defensin levels were also correlated with skin fibrosis suggesting a role in the pathogenesis of fibrosis. An in vitro study investigated effects of alpha defensin in lung tissue that it enhanced expression of IL-8 in epithelial cells as well as transforming growth factor-beta and vascular endothelial growth factor expressions in lung fibroblasts [28]. Another study confirmed that alpha-defensin contribute neutrophilic lung inflammation via inducing synthesis of a strong neutrophil chemoattractant, IL-8, in airway epithelial cells [29].

In a previous study, Kuzumi et al. reported decreased serum beta-2 defensin levels in SSc patients which was highly dependent on duration of disease [30]. In that study they found significant associations between beta- 2 defensin and telangiectasia, pitting scars and digital ulcers suggesting its contribution to pathogenesis of vasculopathy observed in SSc. However they did not find an association with lung involvement [30]. In our study, we did not find a difference for beta- 1 and beta- 2 defensin levels in SSc patients compared to healthy subjects. Discrepancy between results of studies can be explained by differences in clinical manifestations of patients and duration of disease.

We have several limitations in our study. First, we did not assess serum cytokine levels in SSc patients which might demonstrate association between defensins and inflammatory and fibrotic mediators. Second, number of SSc patients are relatively small and inclusion of more patients might reveal better results.

\section{Conclusions}

In conclusion, serum alpha defensin concentrations are increased in SSc patients and associated with lung involvement and skin fibrosis. Further studies involving more patients and detailed molecular tests are needed to determine whether these molecules contribute to the course and pathogenesis of systemic sclerosis.

\section{Abbreviations \\ ANA: Anti-nucleer antibody; AMP: Antimicrobial peptides; DLCO: Diffusing Capacity of Lung for Carbon Monoxide test; ECG: Elektrocardiografi; \\ Echo: Echocardiografi; FEV-1: Functional Expiratory Volume; FVC: Force vital capacity; hBD - 1.2.3: Human Beta defensins 1.2.3; HRCT: High resolution computerized tomography; HNP: Human Neutrophil Peptides; Rf: Raynaud Fenomeni; PAH: Pulmoner Arteryel Hypertension; RFT: Respiratory function test; SSk: Sistemik Skleroz; SP-D: Surfaktan protein; COP: Cryptogenic \\ organized pneumonia; PAP: Pulmonary alveolar proteinosis; NSIP: Idiopathic nonspecific interstitial pneumonia; AMP: Antimicrobial peptides; ARDS: Acute respiratuar distress syndrome; FMF: Familial Mediterranean fever; IPF: Idiopatic pulmonary fibrosis; ELISA: Enzyme-linked immunosorbent assay; RNP: Ribonucleoprotein; IQR: Interquartile range; ACA: Anti-centromere; ESR: Erythrocyte sedimentation rate; CRP: C-reactive protein}

\section{Acknowledgements}

The authors would like to thank the patients and healthy volunteers.

\section{Authors' contributions}

Gedik T.E. and Tufan A. designed the study. Gedik T.E., Kucuk H., Goker B., Haznedaroglu S., Varan O, Ozturk M.A., Tufan A., Pasaoglu H., Pasaoglu O.T. collected the data. Gedik T.E. and Tufan A. performed the statistical analysis. Gedik T.E., Kucuk H., Goker B., Haznedaroglu S., Varan O, Ozturk M.A., Tufan A. interpreted and discussed the results. Gedik T.E. and Tufan A. wrote the paper. Gedik T.E., Kucuk H., Goker B., Haznedaroglu S., Varan O, Ozturk M.A., Tufan A., Pasaoglu H., Pasaoglu O.T. contributed to the final version of the manuscript. All authors have reviewed and approved the final version of the article, including the authorship list. 


\section{Funding}

This research was supported by Gazi University Scientific Research Projects Unit with project number SBE-01 / 2015-41. The funding sources had no involvement in the study design, collection, analysis and interpretation of data, writing of the report, and the decision to submit the article for publication.

\section{Availability of data and materials}

The datasets used and/or analysed during the current study are available from the corresponding author on reasonable request.

\section{Ethics approval and consent to participate}

This study was approved by the Ethics Committee Board of Gazi University Faculty of Medicine.

- Applications No: 28.09.2015/3. All subjects signed the informed consent form. The study was conducted in accordance with the principles of the Declaration of Helsinki.

\section{Consent for publication}

Not applicable.

\section{Competing interests}

The authors declare that they have no competing interests.

\section{Author details}

${ }^{1}$ Department of Internal Medicine, Gazi University Faculty of Medicine, Ankara, Turkey. ${ }^{2}$ Department of Internal Medicine, Division of Geriatrics, Istanbul University-Cerrahpasa, Cerrahpasa Faculty of Medicine, Istanbul, Turkey. ${ }^{3}$ Department of Internal Medicine, Division of Rheumatology, Gazi University Faculty of Medicine, Ankara, Turkey. ${ }^{4}$ Department of Biochemistry, Gazi University Faculty of Medicine, Ankara, Turkey.

Received: 18 August 2020 Accepted: 8 December 2020

Published online: 22 December 2020

\section{References}

1. Almeida C, Almeida I, Vasconcelos C. Quality of life in systemic sclerosis. Autoimmun Rev. 2015;14(12):1087-96.

2. Budd RC, Mclnnes IB, Ruddy S. Kelley's textbook of rheumatology. 8th ed. Philadelphia: Elsevier W.B. Saunders; 2008.

3. Oppenheim JJ, Biragyn A, Kwak LW, Yang D. Roles of antimicrobial peptides such as defensins in innate and adaptive immunity. Ann Rheum Dis. 2003; 62(Suppl 2):ii17-21.

4. Schneider JJ, Unholzer A, Schaller M, Schäfer-Korting M, Korting HC. Human defensins. J Mol Med (Berl). 2005;83(8):587-95.

5. Rivas-Santiago B, Hernandez-Pando R, Carranza C, Juarez E, Contreras JL, Aguilar-Leon D, et al. Expression of cathelicidin LL-37 during mycobacterium tuberculosis infection in human alveolar macrophages, monocytes, neutrophils, and epithelial cells. Infect Immun. 2008;76(3):935-41.

6. Prado-Montes de Oca E. Human beta-defensin 1: a restless warrior against allergies, infections and cancer. Int J Biochem Cell Biol. 2010;42(6):800-4.

7. Kolls JK, McCray PB Jr, Chan YR. Cytokine-mediated regulation of antimicrobial proteins. Nat Rev Immunol. 2008;8(11):829-35.

8. Vordenbäumen S, Fischer-Betz R, Timm D, Sander O, Chehab G, Richter J, et al. Elevated levels of human beta-defensin 2 and human neutrophil peptides in systemic lupus erythematosus. Lupus. 2010;19(14):1648-53.

9. Sthoeger ZM, Bezalel S, Chapnik N, Asher I, Froy O. High alpha-defensin levels in patients with systemic lupus erythematosus. Immunology. 2009; 127(1):116-22.

10. Liang W, Diana J. The dual role of antimicrobial peptides in autoimmunity Front Immunol. 2020;11:2077. https://doi.org/10.3389/fimmu.2020.02077 PMID: 32983158; PMCID: PMC7492638.

11. Hizal M, Bruni C, Romano E, Mazzotta C, Guiducci S, Bellando Randone S, et al. Decrease of LL-37 in systemic sclerosis: a new marker for interstitial lung disease? Clin Rheumatol. 2015;34(4):795-8.

12. Kreuter A, Hyun J, Skrygan M, Sommer A, Bastian A, Altmeyer P, et al. Ultraviolet A1-induced downregulation of human beta-defensins and interleukin-6 and interleukin-8 correlates with clinical improvement in localized scleroderma. Br J Dermatol. 2006;155(3):600-7.

13. van den Hoogen F, Khanna D, Fransen J, Johnson SR, Baron M, Tyndall A, et al. 2013 classification criteria for systemic sclerosis: an American college of rheumatology/European league against rheumatism collaborative initiative. Ann Rheum Dis. 2013;72(11):1747-55.

14. LeRoy EC, Black C, Fleischmajer R, Jablonska S, Krieg T, Medsger TA Jr, et al. Scleroderma (systemic sclerosis): classification, subsets and pathogenesis. J Rheumatol. 1988;15(2):202-5.

15. Yang D, Chertov O, Oppenheim JJ. Participation of mammalian defensins and cathelicidins in anti-microbial immunity: receptors and activities of human defensins and cathelicidin (LL-37). J Leukoc Biol. 2001;69(5):691-7.

16. Oppenheim JJ, Yang D. Alarmins: chemotactic activators of immune responses. Curr Opin Immunol. 2005;17:359-65.

17. Ganz T. Extracellular release of antimicrobial defensins by human polymorphonuclear leukocytes. Infect Immun. 1987;55:568-57.

18. Tamiya H, Tani K, Miyata J, Sato K, Urata T, Lkhagvaa B, et al. Defensins- and cathepsin G-ANCA in systemic lupus erythematosus. Rheumatol Int. 2006; 27(2):147-52.

19. Tufan A, Mercan R, Pasaoglu OT, Pasaoglu H, Ozturk MA, Goker B, et al. Serum antimicrobial peptides in patients with familial Mediterranean fever. Peptides. 2014;57:17-9.

20. Jansen PA, Rodijk-Olthuis D, Hollox EJ, Kamsteeg M, Tjabringa GS, de Jongh GJ, et al. Beta-defensin-2 protein is a serum biomarker for disease activity in psoriasis and reaches biologically relevant concentrations in lesional skin. PLoS One. 2009:4(3):e4725.

21. Lande R, Chamilos G, Ganguly D, Demaria O, Frasca L, Durr S, et al. Cationic antimicrobial peptides in psoriatic skin cooperate to break innate tolerance to self-DNA. Eur J Immunol. 2015;45:203-13. https://doi.org/10.1002/eji. 201344277.

22. Morizane S, Yamasaki K, Muhleisen B, Kotol PF, Murakami M, Aoyama Y, et al. Cathelicidin antimicrobial peptide LL-37 in psoriasis enables keratinocyte reactivity against TLR9 ligands. J Invest Dermatol. 2012;132: 135-43. https://doi.org/10.1038/jid.2011.259.

23. Bokarewa Ml, Jin T, Tarkowski A. Intraarticular release and accumulation of defensins and bactericidal/permeability-increasing protein in patients with rheumatoid arthritis. J Rheumatol. 2003:30:1719-24.

24. Ashitani J, Mukae H, Arimura Y, Sano A, Tokojima M, Nakazato M. High concentrations of alpha-defensins in plasma and bronchoalveolar lavage fluid of patients with acute respiratory distress syndrome. Life Sci. 2004; 75(9):1123-34. https://doi.org/10.1016/j.lfs.2004.01.028.

25. Sakamoto $N$, Ishimatsu $Y$, Kakugawa T, Yura H, Tomonaga M, Harada T, et al. Elevated plasma a-defensins in patients with acute exacerbation of fibrotic interstitial pneumonia. Respir Med. 2015 Feb;109(2):265-71.

26. Mukae $H$, liboshi $H$, Nakazato M, Hiratsuka T, Tokojima M, Abe K, et al. Raised plasma concentrations of alpha-defensins in patients with idiopathic pulmonary fibrosis. Thorax. 2002 Jul;57(7):623-8.

27. Mukae $H$, Ishimoto $H$, Yanagi S, Ishii $H$, Nakayama S, Ashitani J, et al. Elevated BALF concentrations of alpha- and beta-defensins in patients with pulmonary alveolar proteinosis. Respir Med. 2007;101(4):715-21.

28. Amenomori M, Mukae H, Ishimatsu Y, Sakamoto N, Kakugawa T, Hara A, et al. Differential effects of human neutrophil peptide-1 on growth factor and interleukin-8 production by human lung fibroblasts and epithelial cells. Exp Lung Res. 2010;36(7):411-9.

29. Sakamoto N, Mukae H, Fujii T, Ishii H, Yoshioka S, Kakugawa T, et al. Differential effects of alpha- and beta-defensin on cytokine production by cultured human bronchial epithelial cells. Am J Physiol Lung Cell Mol Physiol. 2005;288(3):L508-13.

30. Kuzumi A, Yoshizaki A, Fukasawa T, Ebata S, Miura S, Yoshizaki A, et al. Serum levels of human $\beta$-defensin 2: possible association with fibrosis and vasculopathy in patients with systemic sclerosis. J Eur Acad Dermatol Venereol. 2019;33(7):e272-4.

\section{Publisher's Note}

Springer Nature remains neutral with regard to jurisdictional claims in published maps and institutional affiliations. 